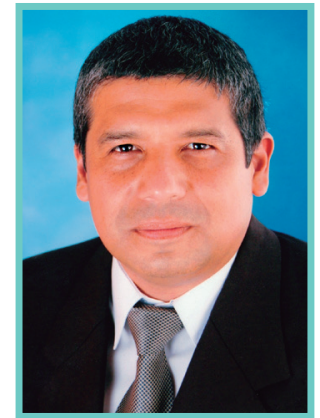

Renatto Merino Solari

\title{
LA EXPERIENCIA COMPRENSIVA COMO FUNDAMENTO DE UNA EDUCACIÓN INTERCULTURAL CRÍTICA
}

\section{RESUMEN}

Desde la década de 1990, el discurso de la interculturalidad ha cobrado importancia en los países de la región, al punto de lograr su reconocimiento constitucional y tener presencia en las políticas educativas. Esta oficialización pone a la interculturalidad frente al reto de sostener desde el interior del sistema el originario espíritu transformador que la impulsa. Sin embargo, en nuestro sistema educativo parece predominar la aplicación de una interculturalidad funcional que no ha producido los cambios esperados. El presente artículo argumenta acerca de la posibilidad de volver concreta una interculturalidad crítica desde el ámbito educativo. Propone a la experiencia comprensiva como el acto fundante de esta pedagogía. Y lo hace a partir de las ideas que sobre la educación desarrolló José María Arguedas.

\section{PALABRAS CLAVE}

Cultura, escuela, interculturalidad crítica, experiencia comprensiva, educación intercultural.

\section{ABSTRACT}

Since the 1990s, the discourse of interculturality has become important in the countries of the region, to the point of achieving constitutional recognition and presence in educational policies. This formalization interculturality makes the challenge of sustaining the system from within the original spirit that drives transformer. However, in our educational system it seems to dominate the use of functional interculturality that has not produced the expected changes. This essay argues about the possibility of returning a critical intercultural concrete from education. It proposes a comprehensive experience as the founding act of this pedagogy. And it does from ideas developed education José María Arguedas.

\section{KEYWORDS}

Culture, school, critical interculturality, comprehensive experience, intercultural education.
I.

El Perú es un país de gran diversidad cultural. La convivencia, vista en perspectiva histórica, ha tenido como ejes articuladores el mestizaje y la aculturación. Ambos fenómenos han facilitado la dominación de los pueblos originarios $y$ su asimilación a las formas dominantes del ser social. Por otra parte, también han ampliado la complejidad de nuestro escenario social, pues la permanente y asimétrica convivencia ha producido, entérminos culturales, pérdidas, incorporaciones $y$ resignificaciones tanto de los elementos propios como de los incorporados. De tal manera que las diferentes culturas que interactúan en nuestra realidad expresan una muy variada gama de matices culturales. 
En un contexto tan complejo es válido preguntarse cómo debe ser la educación. Desde sus inicios en el período colonial, el sistema educativo formal ha cumplido la función de asimilar a las poblaciones originarias en función de los intereses del Estado monocultural. En este momento se estructuraron las bases de la pedagogía contemporánea imponiéndose un modelo pedagógico en el cual se reemplazó al quechua por el castellano, a los amautas por el sacerdote, a la oralidad por la escritura y el libro (Gonzáles y Galdo, 1984). En otros términos, se instaló una concepción educativa basada en una nueva forma de producir y transmitir conocimientos que excluía los saberes de las poblaciones indígenas andinas y amazónicas. Al comenzar la etapa republicana, esta estructura de saber/poder continuó en sus fundamentos. Como ocurrió con los aspectos políticos, económicos y sociales, el molde colonial también se mantuvo en el sistema educativo. Durante el siglo XX, la educación ha sido uno de los principales recursos utilizado por las élites políticas e intelectuales con el objetivo de lograr la integración nacional. Pero los intentos se han llevado a cabo a partir de políticas homogeneizadoras que han tenido como objetivo la aculturación de las poblaciones indígenas. Como señala Montoya (2010), el ideal en el Perú ha sido el Estado monocultural; es decir, un estado, una nación, una cultura, una religión, una lengua y, como consecuencia, una escuela para todos.

Bajo este paradigma, la diversidad cultural y lingüística solo podía ser vista como un problema. Ha tomado mucho tiempo para que esta concepción cambie y la diversidad sea asumida como una posibilidad. Actualmente, la interculturalidad se encuentra incorporada en la esfera educativa oficial y se ha avanzado un tramo importante desde los iniciales esfuerzos por una escuela bilingüe hasta el reconocimiento de la interculturalidad como un principio rector del sistema educativo del país. Sin embargo, desde el discurso oficial parece haber predominado una noción de interculturalidad funcional que no ha producido cambios sustanciales (Trapnell y Zavala, 2013).

Este artículo es una reflexión acerca de la educación intercultural desde la perspectiva de la interculturalidad crítica. Se propone discutir las condiciones sobre las cuales es posible volver concreta una propuesta educativa que supere los límites de la interculturalidad funcional y contribuya a disolver las relaciones asimétricas de poder.

Las ideas que expondremos tienen como motivación la experiencia pedagógica de José María Arguedas, quien fue profesor durante 30 años, primero en escuelas de Cusco y Lima; luego en las universidades de San Marcos, Enrique Guzmán y Valle y Agraria. Este rol de Arguedas todavía no ha sido suficientemente explorado por los estudiosos; sin embargo, se trata de una experiencia muy valiosa, que Arguedas supo enriquecerconsustrabajosdecampo,legándonos un conjunto de descripciones e interpretaciones muy interesantes y todavía vitales acerca del fenómeno educativo en el Perú. En términos antropológicos, destaca el haber desentrañado el valor que las poblaciones andinas le atribuyeron a la educación. La educación ha sido el gran mito movilizador del siglo XX y Arguedas lo percibió apropiadamente.

En esta ocasión no vamos a realizar un análisis formal del pensamiento educativo de Arguedas. De lo que se trata es de tomar sus ideas como motivación para generar una reflexión productiva; y lo hacemos con bastante libertad buscando establecer un diálogo propiciatorio de argumentos.

\section{II.}

Los escritos antropológicos y literarios de Arguedas tienen el mérito de expresar de manera diáfana el sentir de los sujetos, así como haber configurado una visión de conjunto acerca de nuestra sociedad. Se intersectan en su obra la sensibilidad del individuo capaz 
de experimentar las experiencias de los otros como propias y la profundidad del análisis que permite una visión del país como proyecto de nación. Es probable que esta particular sensibilidad se cultivase a partir de una vida muy rica en experiencias diversas que presentó episodios dolorosos y sufrientes, pero que vista en conjunto lo nutrió de una capacidad especial que le permitió comprender de manera privilegiada los tiempos que le tocó vivir. Como señala Pinilla (1995), la comprensión es un arte que las personas ejercitamos cotidianamente y para cuya práctica Arguedas se encontraba especialmente dotado. No es de extrañar que Flores Galindo (1992) lo considerara un personaje excepcional, pues su vida $y$ obra lograron condensar las tensiones y las preocupaciones de nuestra sociedad.

Su experiencia pedagógica no es ajena a estas consideraciones. Por ello es posible encontrar rastros que nos permiten dar forma, a manera de primeros trazos, a una propuesta pedagógica comprensiva que reconfigure la relación que convencionalmente se ha establecido entre el docente y el estudiante.

Una de las primeras cosas que destaca en Arguedas es su preocupación por el estudiante. En una conferencia realizada en 1965 les dice a los futuros profesores:

"Un maestro no puede formar a sus niños, no puede ponerse en comunicación íntima, cariñosa con ellos si no conoce lo más aproximadamente posible cómo es su espíritu... Es el conocimiento del modo de ser de la persona quizás lo más importante para el educador" (Arguedas 2012b: 68).

Esta preocupación por el otro no es solo cognoscitiva. Además del conocimiento del niño en abstracto, es decir, como individuo perteneciente a una etapa del desarrollo humano que presenta un determinado conjunto de características comunes, en Arguedas se evidencia la preocupación por el niño particular, el niño concreto; el que cotidianamente frente a nosotros nos demanda con su presencia y mirada.

El reto que nos propone el educando es el de la intersubjetividad y esto supone el reconocimiento de la existencia del otro como sujeto. No existe individuo a priori ni en abstracto, su existencia ocurre a partir de una relación intersubjetiva específica.

En la concepción convencional, la relación profesor - estudiante queda definida a través de la transmisión de saberes que realiza el primero hacia el segundo. Aquí uno es sujeto activo y el otro es pasivo. El primero define, planifica, dirige y evalúa el proceso educativo, es el experto, es la autoridad. Acompaña, guía y tiene el control de principio a fin. Al otro solo le queda dar las respuestas esperadas para ser calificado apropiadamente. Esta concepción está centrada en una relación asimétrica entre el docente y el estudiante. Es una relación de poder.

En este sentido, la escuela ha sido un espacio de colonialidad, pues ha reproducido hegemonías y subalternidades legitimando los procesos de dominación y de exclusión social. Históricamente, ha negado al otro la posibilidad de representarse autónomamente $y$, por el contrario, a partir de una relación asimétrica, lo ha construido como analfabeto, ignorante, atrasado, sucio, primitivo, etcétera. Así, la escuela ha funcionado como un espacio de finitud simbólica para el diferente y las diferencias.

La experiencia arguediana cuestiona esta concepción y nos plantea una interacción sujeto - sujeto, en la que el intercambio de experiencias implica un descentramiento del individuo, un desbordamiento de los límites del yo para redefinirse en la interacción. No es posible educar sin intercambio existencial que permite la aceptación y el reconocimiento de ambas partes como sujetos. Esta experiencia 
es fundamental para que el maestro pueda acceder a lo que Arguedas denominaba el "alma infantil", es decir, acercarse a la forma de sentir y de pensar del educando.

En un país como el Perú, por su gran diversidad, esto significa un desafío muy grande para el docente. El estudiante es portador de su historia personal, pero a la vez, en él se encarnan los múltiples procesos socioculturales que van cambiando el rostro del país. En este sentido, el aula resulta un espacio múltiple, heterogéneo y conflictivo, por tanto, deviene en realidad compleja. Las alteridades que se generan son consecuencia de la confluencia de múltiples perspectivas que se acercan, se distancian, se enfrentan y se mezclan transformando a todos los participantes. El aula funciona así como un espacio para la realización de lo que Hopenhayn (2002) denomina la utopía transcultural. Es decir, la posibilidad de redefinirnos a partir de un "vuelo existencial" que nos desplaza desde la mismidad hacia la otredad para hacernos regresar al yo luego de habitar en la subjetividad de los otros. Se trata de una autocreación transcultural como expresión de múltiples síntesis.

La relación de alteridad que se genera en el acto educativo tiene implícita la necesidad no solo de conocer sino fundamentalmente de comprender al otro. Conocer al otro es definirlo objetivamente desde sus características para clasificarlo y calificarlo. Lo que se requiere es un maestro que vaya más allá de los límites cognoscitivos y sea capaz de comprender al estudiante en su singularidad, tal cual es, como sujeto concreto que se está construyendo permanentemente y que constituye una promesa de humanización.

Este sujeto no se encuentra descontextualizado, forma parte de una cultura y tradición que, además, son dinámicas. Por ello, la necesidad de comprender el contexto sociocultural en el cual está inserto es primordial. Como profesor en el Colegio Nacional "Mateo Pumaccahua" de Sicuani, Cusco (2012d), Arguedas, hizo que sus alumnos estudiaran la región en donde funcionaba el colegio y los pueblos de los que procedían como una forma de conocer su propia realidad material y espiritual. La necesidad de enraizar la escuela en su contexto, en la cultura del lugar, es fundamental en todo proceso educativo; este contacto directo le permite al estudiante descubrir las riquezas de su cultura, valorarlas y afirmar su identidad.

En su texto sobre el niño indio (2012a), Arguedas nos muestra la importancia de conocer las creencias mágicas y religiosas así como los factores socioeconómicos que influyen en la vida de los niños de las comunidades:

"El niño que nace y crece en un mundo en que la vida humana está relacionada y depende de la vida consciente de las montañas, de las piedras, insectos, ríos, lagos y manantiales, se forma considerando el mundo y su propia existencia de una manera absolutamente diferente que el niño de una ciudad, en que solo el ser humano está considerado como animado por un espíritu" (2012ã. 234). ${ }^{1}$

El niño andino amazónico por las influencias de su entorno ambiental y cultural va a desarrollar una forma de pensar y de actuar, es decir, un ser social diferente al del niño que se desarrolla en las grandes ciudades. Sin embargo, la experiencia urbana y la educación escolar introducían nuevos valores que estaban haciendo cambiar dicha concepción. Por ello, nos dice Arguedas, que el niño indio afronta una situación contradictoria. Por una parte, los ancianos le enseñaban que la naturaleza tiene carácter sagrado y debía ser venerada; por otra parte, los jóvenes ya no estaban muy

1 Las cursivas corresponden al texto citado. 
dispuestos a continuar las tradiciones y para ellos la naturaleza solo representaba un "monte seco".

Esta realidad que cotidianamente vivía el niño andino era la expresión del conflicto cultural que atravesaba el país y que, en gran medida, se mantiene hasta nuestros días. La escuela no fue capaz de entenderlo convirtiéndose en una de las principales herramientas de aculturación de las poblaciones originarias. Muchos maestros tampoco entendieron la dramática situación de los niños pertenecientes a comunidades y pueblos originarios. Por el contrario, hicieron suyo el discurso homogeneizador y excluyente de las élites políticas e intelectuales asumiendo activamente el rol de agentes de la modernidad occidental.

Al respecto, resulta interesante la experiencia descrita por Kapsoli (1986):

"Cuando estudiábamos en la Escuela Prevocacional de Pomabamba (Ancash) fuimos testigos de una 'ingeniosa' represión del quechua. El profesor impuso un castigo psicológico, con efectos violentos de apocamiento. Los alumnos sorprendidos hablando quechua eran conducidos al centro de la Plaza con una corona de papel rotulado: "El Rey de los Quechueros". Las víctimas, generalmente alumnos de las estancias y caseríos, eran pasto de burla y hazmerreír de los citadinos" (Kapsoli 1986: 13). ${ }^{2}$

En otro momento y lugar, Montoya (1990) recoge el siguiente testimonio de una escuela:

"Si hablaba alguna palabra en Kichua, el profesor me hacía estar de rodillas con piedras debajo... Hablar castellano, vestir pantalón y camisa son las condiciones para ser gente... Me había tenido que frotar mi cara con arena para sangrar. Estabapintada, no podía pasar a la escuela" (Montoya 1990: 20). ${ }^{3}$

Es más que medidas represivas "ingeniosas". La acción del maestro, en ambos casos, se sustenta en una estructura de poder y en una episteme de raíz colonial que ha permitido la exclusión histórica de las poblaciones originarias, o peor aún, una matriz colonial que ha incluido para disolver, para desnaturalizar al otro. Es evidente que la escuela no ha cumplido con su rol liberador. Es cierto que permitió el ascenso social de algunos sectores, pero el costo ha sido muy grande: negar la condición del ser.

¿Cuál es la educación que un país como el Perú requiere? Probablemente no exista respuesta única y sea necesario inventar formas otras de educar en correspondencia con los espacios particulares y las microhistorias:

"En un país tan complejo como el nuestro, tan lleno de contradicciones culturales, de creencias distintas, la misión del maestro es realmente aquí, una misión muy difícil; porque en el Perú la educación no se resuelve mediante el método sino mediante el conocimiento de la cultura, de las costumbres de cada pueblo, porque somos un país muy mezclado, un país mestizo en cuanto a creencias, en cuanto a concepciones morales, políticas; en fin, somos un país que constituye una mezcla que todavía no ha acabado de definirse" (2012b: 74).

Para Arguedas el problema de la educación no es un tema exclusivamente pedagógico; por el contrario, es esencialmente un problema cultural: "la educación se resuelve mediante el conocimientodelacultura”.Aquíestáimplícitoel problema cardinal de nuestra institucionalidad: tener una educación monocultural en un país

2 Las cursivas y comillas corresponden al texto citado.

3 Las cursivas corresponden al texto citado. 
de grandes diferencias culturales. Por esta razón la escuela no llegó a tocar las fibras más sensibles del tejido social. Ha sido, en gran medida, una institución desarraigada, que no sintonizó con las necesidades y demandas de las mayorías, y esto ha sido más grave en el caso de las poblaciones originarias.

Un país "que todavía no ha acabado de definirse" y que seguramente nunca lo conseguirá, pues las sociedades heterogéneas son realidades de transición y de intercambios permanentes, requiere con urgencia de una educación intercultural universal, no en el sentido de homogénea, sino de para todos. Una educación intercultural sectorizada corre el riesgo de convertirse en parte del problema y, además, es negarle a los peruanos (indígenas, afros, criollos y mestizos de toda índole) el conocimiento profundo de lo más valioso que tienen: la diversidad humana.

Por otra parte, la práctica pedagógica intercultural requiere de docentes con capacidad de comprensión superlativa, que sean portadores de lo que podríamos denominar la "sensibilidad arguediana":

"Si no sabemos qué es lo que piensan acerca de dios, de cómo es dios, de por qué es dios, de para qué es dios, para qué vive el hombre y por qué vive el hombre y hacia dónde se dirige el hombre, si no sabemos lo que el pueblo piensa acerca de estos problemas ¿cómo podemos educarlos? La educación no consiste solamente en dar instrucción e instrucción en una forma bastante rutinaria; hay que darla siempre de acuerdo con los incentivos característicos espirituales que en cada comunidad mueven al hombre" (2012b: 73).

Esta es la actitud que se requiere en un país que se presenta como una mistura de creencias y concepciones morales y políticas. Comprender el sentido y el significado que mueven el pensar, el actuar y el sentir de los estudiantes es asumir la sensibilidad arguediana como fundamento de nuestra experiencia docente. Este enlazamiento entre biografía personal y devenir histórico es fundamental en la práctica pedagógica intercultural.

\section{III.}

Los discursos acerca de la interculturalidad son diversos. La interculturalidad es reivindicada por grupos sociales históricamente excluidos que plantean la necesidad de nuevas formas de vida social. Pero también es un discurso al cual recurren estados y poderes transnacionales defensores del orden establecido. Para sintetizar el punto, y de acuerdo con Walsh (2010), es posible plantear la existencia de tres perspectivas acerca de la interculturalidad. La perspectiva relacional, cuyo énfasis se encuentra en la experiencia de intercambio, en las relaciones que históricamente han establecido las culturas y que de por sí configuran una situación de interculturalidad. La perspectiva funcional que, partiendo de reconocer las diferencias culturales, busca incorporarlas al sistema social hegemónico. La perspectiva crítica, aquella que sin desconocer la importancia de las diferencias culturales, su punto de partida es el cuestionamiento al predominio de la estructura de poder colonial que sustenta la hegemonía de la matriz civilizatoria occidental. La interculturalidad crítica es fundamentalmente política.

Las dos primeras en lo esencial no pretenden una alteración de las estructuras de dominación y muy comúnmente terminan siendo funcionales al ejercicio del poder hegemónico. Por el contrario, la interculturalidad crítica apunta necesariamente a la transformación de las estructuras y de las relaciones sociales para reescribir la historia universal a partir de las múltiples concepciones del sery de la vida. Es, por esto mismo, un proyecto en curso cuyo origen está en las discusiones políticas que proponen los movimientos sociales contra-hegemónicos y exige una acción de transformadora (Walsh, 2012). 
Una interculturalidad crítica requiere una educación transformadora. En este momento no estamos en condiciones de definirla, pero sí podemos proponer algunas coordenadas que orienten en su construcción.

Algo que debemos comenzar considerando es que no es posible plantear una pedagogía crítica. Deben ser múltiples pedagogías y el énfasis debe estar en las prácticas particulares, en las experiencias concretas de cada realidad en la que se presenta el acto educativo. No existe una historicidad tal como Occidente ha impuesto a todos los pueblos, sino una pluralidad de historias que no necesariamente coinciden en la línea del progreso y del desarrollo que ha trazado la historia universal. El reto de las pedagogías interculturales se encuentra, precisamente, en la reconstrucción de estos tiempos históricos como base para el diálogo entre culturas.

Es necesario construir experiencias educativas que sean capaces de cuestionar dos hegemonías que históricamente han generado relaciones asimétricas entre las culturas. Por una parte, las estructuras de poder construidas a partir del predominio de Occidente que asume una supuesta superioridad civilizatoria $y$, por otra, la preeminencia de la producción de los conocimientos científicos - tecnológicos que subordinan otras formas de generar saberes y de relacionarse con el entorno. En la medida que esto no ocurra difícilmente podrá establecerse vínculos que revaloren las historias locales y las epistemologías oscurecidas. La educación como espacio de finitud debe dar paso a la educación como espacio de encuentro de horizontes.

En este sentido, el reto para la educación intercultural es superar lo que Arguedas (2012c) denominaba la "actitud del conquistador"; es decir, el supuesto de la superioridad de ciertas razas y culturas sobre otras, imaginario con el cual el conquistador legitimó su dominación.
Esta actitud hace imposible ver a los otros como iguales, así como reconocer sus capacidades creativas y logros presentes. Asimismo, es la mayor evidencia de que la matriz colonial se encuentra aún vigente tanto en las prácticas como en las estructuras mentales de las personas:

"La diversidad cultural y lingüística del Perú está marcada por un hecho esencial: la dominación de la cultura occidental criolla sobre las 58 culturas restantes del país. Los conquistadores y vencedores de ayer y hoy creyeron y creen, que su cultura fue $\mathrm{y}$ es 'superior' a las otras e impusieron la suya como modelo o ejemplo a seguir. 'Cristianizar' y 'civilizar' son las dos categorías fundamentales en las que se expresa este complejo proceso de dominación cultural y lingüística" (Montoya, 1990: 23). ${ }^{4}$

Las pedagogías transformadoras están gestándose desde los espacios intersticiales de las sociedades, especialmente en las multiétnicas. Es decir, se están generando en los márgenes del sistema, más allá de las instituciones formales, en las experiencias de las comunidades que educan desde la praxis social y política. Ellas “integran el cuestionamiento y el análisis crítico, la acción social transformadora, pero también la insurgencia e intervención en los campos del poder, saber y ser, y en la vida" (Walsh, 2012: 15).

Por su carácter innovador es un proceso germinal, heterogéneo, disperso y en gran medida utópico. Existen intentos por incorporarla al régimen político lo cual podría poner en riesgo su carácter cuestionador. ¿Qué ocurre con un movimiento que emergiendo desde las bases logra ingresar al sistema?; ies posible disolver la matriz colonial desde el espacio político y educativo formal?

4 Las cursivas y comillas corresponden al texto citado. 
La incorporación de la interculturalidad a los procesos políticos oficiales reconfigura el escenario para ambas partes. De manera muy esquemática podemos señalar que en procesos políticos transformadores la interculturalidad tendrá mayores espacios para, desde la educación, acompañar los cambios propuestos; por el contrario, en estructuras políticas conservadoras, la incorporación de la interculturalidad en la educación puede devenir en nuevas metodologías asimilacionistas y en un discurso sin mayor correlato con la praxis que termina folklorizando las diferencias culturales. Para desarrollar prácticas transformadoras es necesario que los imaginarios sean reconstruidos; sin embargo, esta es una experiencia que no puede emerger de la relación educativa convencional por ser unidireccional. Pensamos que el hecho de escapar a los límites

\section{REFERENCIAS}

Arguedas, J. (2012a). Algunas observaciones sobre el niño indio actual y los factores que modelan su conducta. En: José María Arguedas. Obra antropológica. Tomo 7. Lima: Horizonte. pp. 232-239.

(2012b). El cuento folklórico como fuente para el estudio de la cultura. En: José María Arguedas. Obra antropológica. Tomo 7. Lima: Horizonte. pp. 68-74.

(2012c). Un método para el caso lingüístico del indio peruano. En: José María Arguedas. Obra antropológica. Tomo 1. Lima: Horizonte. pp. 376-382.

(2012d). Pumaccahua. Trabajos de los alumnos del Colegio Nacional de Sicuani bajo la dirección de José María Arguedas. En: José María Arguedas. Obra antropológica. Tomo 1. Lima: Horizonte. pp. 225-260.

Flores Galindo, A. (1992). Dos ensayos sobre José María Arguedas. Lima: SUR.

Gonzales, E. y Galdo, V. (1984). Procesos e Instituciones. Historia de la Educación en el Perú. Historia del Perú, Tomo X. Lima: Juan Mejía Baca.

Hopenhayn, M. (2002). La aldea global entre la utopía transcultural y el ratio mercantil: paradojas de la globalización cultural. En: Carlos I. Degregori y Gonzalo Portocarrero (editores). Cultura y Globalización. Lima: Red de lo oficial es la mayor riqueza del movimiento y lo dota de un potencial ilimitado.

Finalmente, y al margen de matices e intensidades, una educación intercultural crítica deberá tener como punto de partida la experiencia comprensiva que emerge de la relación intersubjetiva. Se trata de estar abiertos a una vivencia de exteriorización que nos haga salir de la mismidad desplazándonos hacia la otredad y luego volver al yo en una ida y vuelta que no puede ser solo autoreferencial, sino también, y fundamentalmente, relacional. El momento en el cual asumimos que el otro es un sujeto y estamos dispuestos a iniciar juntos la construcción de experiencias a partir de una relación de iguales se convierte así en el acto fundante de una pedagogía transformadora, creativa y comprometida.

para el Desarrollo de las Ciencias Sociales en el Perú. pp. $17-36$.

Kapsoli, W. (1986). Nosotros los Maestros. Lima: Horizonte.

Montoya, R. (2010). Porvenir de la cultura quechua en Perú. Desde Lima, Villa El Salvador y Puquio. Lima: UNMSM.

(1990). Por una Educación Bilingüe en el Perú. Reflexiones sobre culturay socialismo. Lima: Cepes, Mosca Azul.

Pinilla, C. (1995). Arguedas y el conocimiento comprensivo. En: Maruja Martínez y Nelson Manrique (editores). Amor y fuego. José María Arguedas 25 años después. Lima: Desco, Cepes. pp. 209-232.

Trapnell, L. y Zavala, V. (2013). Dilemas educativos ante la diversidad, siglos XX-XXI. Colección Pensamiento Educativo Peruano, Volumen 14. Lima: Derrama Magisterial.

Wash, C. (2012). Interculturalidad crítica y pedagogía decolonial: Apuestas desde el i-surgir, re-existir y re-vivir. http://www.saudecoletiva2012.com.br/userfiles/file/ didatico03.pdf Reg. 3 enero de 2015.

(2010). Interculturalidad crítica y educación intercultural. En: Jorge Viaña et al. (Editores). Construyendo Interculturalidad Crítica. La Paz: Instituto Internacional del Convenio Andrés Bello. pp. 75-96. 